\title{
DEEP, AEGIS, \& CATS - Pathfinding Surveys to the Next Generation of Distant Galaxy Stellar Population Research
}

\author{
David C. Koo ${ }^{1}$, DEEP2 $^{2}{ }^{2}$ AEGIS $^{3}$ \& CATS $^{4}$ Teams \\ ${ }^{1} \mathrm{UCO} /$ Lick Observatory, Department of Astronomy and Astrophysics, \\ University of California, Santa Cruz, CA 95064, USA \\ email: koo@ucolick.org \\ ${ }^{2}$ see http://deep.berkeley.edu \\ ${ }^{3}$ see http://aegis.ucolick.org \\ ${ }^{4}$ see http://irlab.astro.ucla.edu/cats/index.shtml
}

\begin{abstract}
DEEP, AEGIS, and CATS are examples of spectroscopic, multiwavelength, and adaptive optics surveys, respectively, that are pushing the frontiers of stellar population studies of distant galaxies. Together, these surveys affirm the unprecedented richness of high quality information that can already be gathered using today's generation of ground and space telescopes. We highlight several results in extracting the stellar and dynamical masses, chemical abundances, ages, and frequency of galactic winds for galaxies at redshifts up to $z \sim 1.4$.
\end{abstract}

Keywords. surveys; galaxies: evolution; galaxies: high redshift; galaxies: kinematics; instrumentation: adaptive optics

\section{What are DEEP and AEGIS?}

DEEP (Deep Extragalactic Evolutionary Probe) was initiated in 1991 through the NSF Science \& Technology Center for Particle Astrophysics (CfPA) to undertake a major spectral survey of faint field galaxies with the forthcoming Keck $10 \mathrm{~m}$ Telescope. The use of DEIMOS (DEep Imaging Multi-Object Spectrograph), commissioned in 2002, divides DEEP into its first two phases. The first (DEEP1) used a pre-DEIMOS lower resolution spectrograph (LRIS) to obtaine 600 spectra as faint as $I \sim 24$ mag (Vogt et al. 2005). These Keck spectra were combined with images from from $H S T$ to study distant galaxies and served as a pathfinder on the technical feasibility, depth, and scientific scope of DEEP2, the flagship among the DEEP surveys.

DEEP2 (Newman et al. 2010) is distinguished from prior faint redshift surveys by its large sample of almost 40,000 galaxies that reach faint enough $\left(R_{A B} \sim 24 \mathrm{mag}\right)$ to access ordinary galaxies at redshifts $z \sim 1$. This survey used 90 nights of Keck time and took one hour long exposures per target. To increase our sample of higher redshift galaxies, we used $B R I$ two-color diagrams to preselect galaxies at redshifts $z>0.7$ for three of DEEP2's four fields. The fourth field, which overlaps the original DEEP1 Groth Strip, but is over 10 times larger and thus dubbed the Extended Groth Strip (EGS), covers 0.5 square degrees which is half that of each of the other three fields, but has no color preselection. The inclusion of redshifts below $z \sim 0.7$ in EGS provides better support for the other surveys under the aegis of the All-wavelength Extended Groth International Survey (AEGIS). An upper limit of $z \sim 1.45$ for all fields is set by the accessibility of [OII] $3727 \AA$ up to the red limit of our spectral range $(\sim 9100 \AA)$. 
DEEP2 is also distinguished by adopting a spectral resolution $R \sim 5000$ which is high enough to measure galaxy rotation curves and kinematic linewidths. Such internal kinematics of galaxies provide a powerful new dimension related to the dynamical masses of galaxies. These kinematics may also be intimately tied to dark matter halo masses, which are the fundamental components of galaxies best understood from numerical simulations of galaxy formation.

DEEP3 is a 27 night Keck DEIMOS survey that is a follow-up of the now-completed DEEP2. DEEP3 aims to increase the sampling density in the EGS region covered by $H S T$ by nearly 3 -fold, to reach bluer limits by doubling the spectral range, and to reach $4 \mathrm{x}$ fainter limits $(R \sim 25.5)$ for subsamples of Chandra, Spitzer, and non-active galaxies. These gains were achieved by reducing the spectral resolution by a factor of two.

\section{More on AEGIS}

From its inception, DEEP2 has been designed to be a magnet for panchromatic surveys. While all four of our DEEP2 fields have various amounts of coverage in the near-IR, mid-IR, and X-ray, DEEP2's EGS field has been receiving the lion's share of very deep multiwavelength coverage that range from X-ray to radio. At present, AEGIS is perhaps the premier region in the sky for deep multiwavelength studies of distant dusty star forming and AGN systems because of its relatively large area with an extensive set of very deep surveys across the full spectrum. While the COSMOS field is larger, the depth of its complementary surveys are generally shallower. The GOODS fields have complementary data of similar or better depth, but are significantly smaller in area. More details of the AEGIS surveys are given in the introductory paper to the AEGIS special issue by Davis et al. (2007). Since then, five major blocks of time have been awarded for AEGIS: 1) $3 \mathrm{x} 600 \mathrm{Ks}$ has been added to the existing $8 \mathrm{x} 200 \mathrm{Ks}$ with Chandra, thus providing three regions of $800 \mathrm{Ks}$ each that can be combined with the $2 \mathrm{Ms}$ single pointings in the two GOODS fields to study faint, rare x-ray sources; 2) 3 hour deep MIPS data from Spitzer has been added to the north-east half of EGS, enabling the study of more distant LIRGs and ULIRGs; 3 ) among the deepest $16 \mu \mathrm{m}$ surveys with AKARI, covering most of the AEGIS field; these data lie in the middle of the large wavelength gap between Spitzer's $8 \mu \mathrm{m}$ of channel- 4 with IRAC and the $24 \mu \mathrm{m}$ of MIPS, 4) 12 -hour pointings with Spitzer IRAC's two bluest cameras being taken during its warm mission; and 5) DEEP3.

\section{Why use DEEP2 \& AEGIS for Stellar Population Studies?}

Regions covered by DEEP and AEGIS are particularly attractive for rich, new stellar population studies of distant galaxies because of their:

1) Faint Depths: DEEP2 has an $R=24.1$ limit while DEEP3 targets reach $R=$ 25.5 , faint enough to reach beyond redshifts $z \sim 1$. This is an era of more intense star formation and AGN activity; galaxies are assembling and converging on their presentday structure and morphology; and many counterparts are expected and detectable with current very deep to deepest X-ray, far-UV, mid-IR, far-IR, sub-mm, and radio surveys.

2) Large Sample Sizes: The 15,000 spectra in EGS expected after DEEP3 is done are enough to yield significant numbers of rare, but important, classes of objects, such AGN's and ULIRGs or those being extreme in mass, metallicity, size, etc. Large samples at high surface density are essential for deriving the dependence of galaxy stellar populations on environment, ranging in spatial scales from close pairs, groups, clusters, and filaments to large voids. Larger samples, moreover, allow more and finer subdivisions in galaxy 
property space in working with stacked spectra (or with stacked images from the suite of complementary surveys) to explore trends and correlations to ultra-deep limits.

3) Spectral Resolution: DEEP2 has a spectral resolution of $\sigma \sim 30 \mathrm{~km} / \mathrm{s}$. This is high enough to measure the internal kinematics of galaxies, thereby providing dynamical masses when inclinations and sizes are known. Such "total" masses can then be compared to photometric estimates of stellar masses from optical, near-IR, and mid-IR photometry. Besides stellar masses, dynamical (halo) masses can also be related to other stellar population properties that evolve, such as luminosities, colors, metallicity, age, star formation rate, etc. With high resolution, velocity differences between galaxies can be measured well enough to detect genuine close pairs, low-mass groups, and the relative density of galaxies in the environment of each galaxy. Such groups and environment measures are expected to be relevant parameters for understanding the evolution of stellar populations. Higher resolution also enables better analyses of stellar population measures (age, metallicity, alpha-element enhancement) and gas ionization conditions and metallicity. Finally, higher spectral resolution yield better estimates of line profile shapes and asymmetries, useful, e.g., to study galactic winds and gas inflows.

4) High S/N and Wide Spectral Range: These translate to improved redshift reliability and completeness as well as the detection of a richer set of absorption and emission lines over a wider redshift range. These complement key stellar population measures from other wavelengths, including star formation $(\mathrm{H} \alpha$ in the near-IR, X-ray, FUV, FIR, and radio luminosities), metallicity (as derived from rest-frame near-IR colors seen with IRAC colors); and age (from broadband spectral energy distributions).

\section{CATS: the Center for Adaptive Optics (CfAO) Treasury Survey}

CATS is an near-IR study of the structural, chemical, star formation, and kinematic evolution of distant field galaxy subcomponents on sub-kpc scales. Using adaptive optics (AO) on an 8-10m class telescope yields diffraction-limits in the near-IR of 0.05 arcsec, which is 3-4 times better than is possible with HSTs $2.4 \mathrm{~m}$ mirror at a similar wavelength, and is a near perfect match to $H S T$ 's optical resolution (see Fig. 3). This resolution corresponds to the sizes of bulges/spheroids, disks, bars, spiral arms, and merger/interaction or lensing signatures at redshifts $z>0.5$. Having such a match between the diffractionlimit of HST in the optical and of Keck in the near-infrared drove the CATS strategy to work in well-studied HST survey fields with ACS images taken in 2 or more filters, including GOODS, COSMOS, GEMS, \& EGS. These regions are highly leveraged by deep Chandra, XMM-Newton, GALEX, and Spitzer data from space and optical, near-IR, submm, and radio data from the ground.

Keck's AO system is presently limited to the near-IR, but this wavelength region nicely complements the optical for the study of stellar populations of galaxy subcomponents, by providing superior penetration through dust, yielding a longer baseline for multicolor analyses of stellar populations, and having higher sensitivity to old stars. For redshift $z \sim 1$ to 2 galaxies, the near-IR measures light that was emitted as restframe optical, including the important $H \alpha$ and [NII] lines for spectroscopy. Keck now routinely provides laser guide star (LGS) AO (Wizinowich et al. 2006), which has opened new doors for the study of distant galaxies. With LGS AO supporting tip-tilt stars as faint as 18th mag within $\sim 60$ arcsec and good characterization of the off-axis PSF, $20 \%$ or more of the area of the well-studied HST galaxy survey fields are now accessible to near-IR AO observations. Prior to LGS AO, only about $1 \%$ was reachable on Keck since much brighter natural guide stars of 12 th mag were needed. Thus much of the pioneering distant galaxy research with Keck AO by, e.g., Larkin et al. (2000) and Glassman et al. (2002), were 
undertaken without having the benefit of adding HST data. With LGS AO, CATS has been able to work routinely with combined HST and Keck data (Steinbring et al. 2004, 2008; Melbourne et al. 2005, 2008, Ammons et al. 2009). CfAO is now at the end of its 10 year life and thus CATS will formally close, but CATS has a left a legacy as a pathfinding demonstration of the power of using AO for distant galaxy science, especially studies of stellar populations.

\section{Science Highlights}

DEEP2 is complete and publicly available (see URL: http://deep.berkeley.edu) and DEEP3 is underway. Combined with AEGIS and CATS, the range of science, as published in over 100 refereed papers, has been wide, with a large fraction addressing the evolution of stellar populations. While many galaxy properties appear on average to correlate with stellar mass, another major theme revealed by DEEP is that galaxy evolution, when examined in more detail, is more complicated. Distant field galaxies of the same mass show diversity - in size, luminosity, structure; are usually composed of several subcomponents (e.g., bulges and disks) which appear to have experienced different star formation and dynamical histories and evolution; and reside in a broad range of environments (pairs, groups, clusters, voids, and other large scale structures) that engage different physical processes that affect their evolution in stellar populations.

The following four examples highlight stellar population studies that exploit DEEP2's spectral resolution and large sample size, AEGIS's panchromatic coverage, and the high spatial resolution and the near-IR of CATS.

\subsection{New Velocity versus Stellar Mass Relation}

The Tully-Fisher relation (TFR) between the luminosity of galaxies and their velocity width is a fundamental scaling relation that constrains galaxy formation and evolution models. Recent local TFR studies focus on stellar or baryonic masses, which are easier to model than luminosity. Distant stellar-mass TFRs have also been studied by, e.g., Conselice et al. (2005) and Flores et al. (2006), but neither group found evidence for evolution to redshifts $z \sim 1$ or 0.6 , respectively. They, like those working with local samples, pruned their sample to exclude morphologically odd galaxies, which comprise an increasing fraction for more distant galaxies.

To showcase our kinematics, we present a study of the stellar mass TFR to redshift $z=1.2$ using a sample of 544 galaxies with strong emission lines, but without any pruning for morphology (Kassin et al. 2007) . Undertaking a TFR study using only rotation velocity $\left(V_{\text {rot }}\right)$, we find (see upper panel of Fig. 1) a large scatter, as had been found previously by ourselves and others. Disturbed, compact, and major merger galaxies are the major contributors to the scatter and they do show apparent evolution with redshift, mainly by having lower velocities for their given stellar masses.

A different story emerges, however, after we adopt a new kinematic estimator, $S_{0.5}$, which is defined as $\sqrt{0.5 V_{\text {rot }}^{2}+\sigma_{g}^{2}}$. This measure, by adding the gas velocity dispersion $\left(\sigma_{g}\right)$, includes disordered or non-circular motions. As shown in the lower panel of Fig. 1 , the TFR using $S_{0.5}$ is remarkably tight over $0.1<z<1.2$. As for evolution, we find none for the TFR's intercept or slope. Since the TFR with $S_{0.5}$ lies so close to the Faber-Jackson relation for nearby early-type galaxies, a physical connection between dynamically unsettled systems and early-type galaxies is suggested. Adding to the credibility of this connection, simulations of major mergers show that throughout the full range of phases and viewing angles and disturbed morphologies, $S_{0.5}$, as would be measured with realistic slit widths, seeing, different position angles, and typical redshifts, 
shows a high level of stability due to the rough anti-correlation between observed $V_{\text {rot }}$ and $\sigma_{g}$ (Covington et al. 2010).
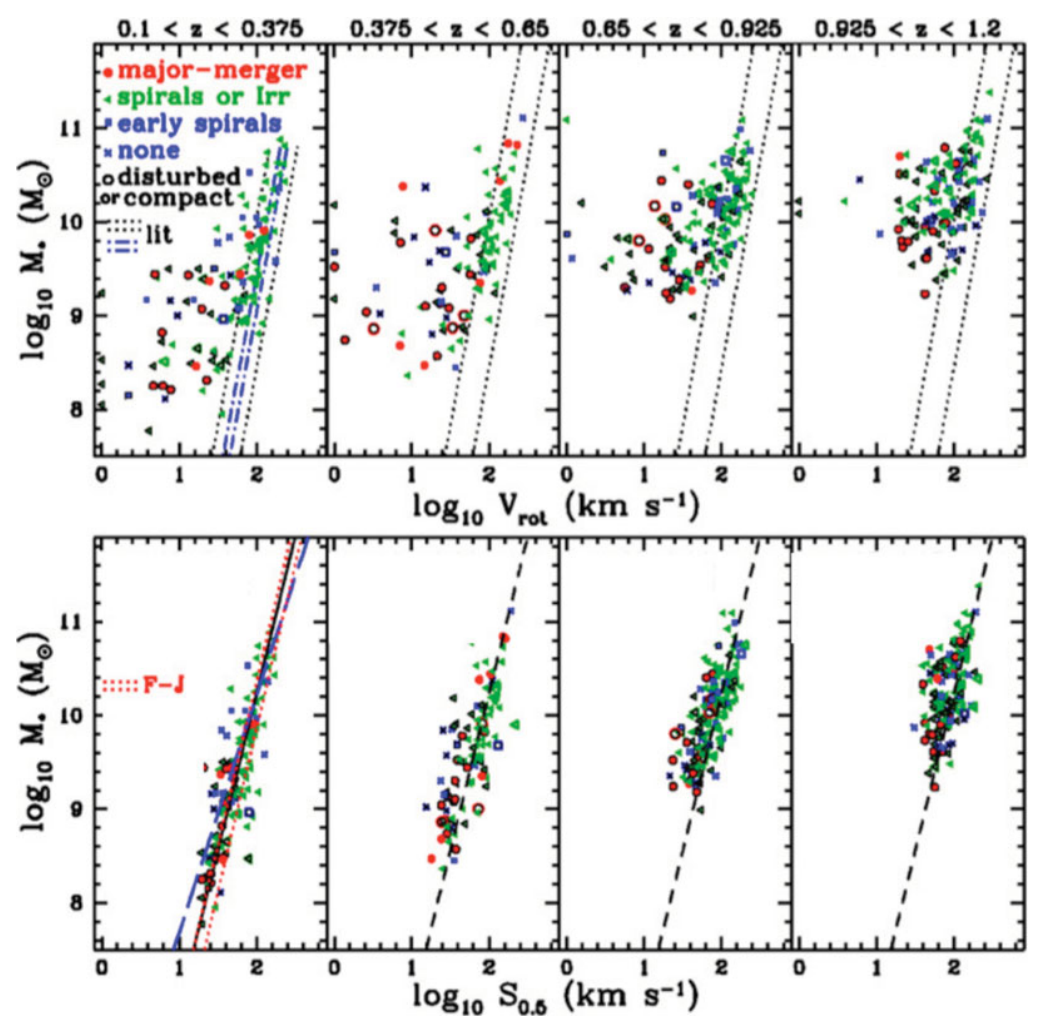

Figure 1. [Upper panels]: The $V_{\text {rot }}$ stellar-mass Tully-Fisher relations (TFR) for $0.1<z<1.2$ in bins of redshift $z$ as indicated at the top and for different morphologies. Left panel stellar mass TFRs from local (dot-dashed) and redshift $z \sim 1$ (dotted) studies delineate rms scatter. [Lower panels:] $S_{0.5}$ yields a much tighter and non-evolving stellar mass TFR to redshifts $z=1.2$. In left panel, solid line shows fit to the lowest redshift data and is repeated as dashed lines at higher redshifts; the local, stellar-mass, Faber-Jackson relation are two dotted lines to delineate rms scatter along with the baryonic mass TFR (dashed). See Kassin et al. (2007) for more details.

\subsection{Distant, Luminous, Metal-Poor, Massive Galaxies - are they Young?}

The metallicity of galaxies is a fundamental property that relates directly to the integral history of star formation and galaxy mass, as well as to gas outflow and infall (Kunth \& Ostin 2000). Metal-poor star-forming systems are unique laboratories for the study of primeval star formation, initial enrichment, feedback, and galaxy mass loss. Work with low-mass, faint dwarf irregulars (Lee et al. 2006), combined with the analysis of 53,000 SDSS galaxies (Tremonti et al. 2004), show that gas-rich star-forming galaxies today exhibit both a very tight luminosity (L) versus metallicity (Z) relation (LZR) and an even tighter stellar mass $(\mathrm{M})$ versus metallicity $(\mathrm{Z})$ relation (MZR).

These relations appear to evolve. As shown in Fig. 2 , at intermediate $(z \sim 0.7)$ to higher redshifts $z>1$, the LZR shifts to brighter galaxies and the MZR to more massive galaxies at a given $[\mathrm{O} / \mathrm{H}]$. Such evolution offers valuable constraints on the chemical enrichment history of galaxies. Locally, closed-box models seem to match the slopes and small dispersions surprisingly well (Lee et al. 2006), though dwarfs appear more depleted in metals than massive galaxies and thus suggest that galactic winds play an important 
role (Tremonti et al. 2004). Similarly, for higher redshift samples, several authors favor closed box models (e.g., Liang et al. 2006, Savaglio et al. 2005, Shapley et al. 2005), while others suggest that winds and outflows are important (Erb et al. 2006).

For distant samples, the metallicities were based on empirical relations among strong

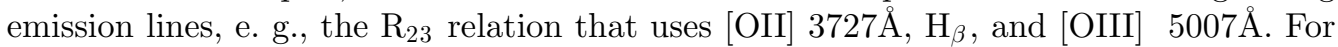
a given value of $\mathrm{R}_{23}$, however, the empirical relation for $[\mathrm{O} / \mathrm{H}]$ has two solutions, one for low and the other for high metallicity. Previous works have chosen the high one. If low metallicity galaxies did exist, they would have been missed.

To bypass such empirical relations and to determine $[\mathrm{O} / \mathrm{H}]$ directly, we undertook a search for the typically weak [OIII] $4363 \AA$ line along with the standard strong lines. We discovered a number of such galaxies at redshifts $0.5<z<0.8$ (Hoyos et al. 2005, 2010). They are luminous and yet metal poor, with measured $[\mathrm{O} / \mathrm{H}]<1 / 3$ solar. Fig. 2 shows that our sample is quite exceptional, with offsets 3 to $10 \sigma$ from the tight local LZR and MZR; these offsets are more than that of even $z \sim 2$ galaxies. Though very rare, roughly 1 in a 1000 and thus requiring a survey as large as DEEP2 to uncover a decent sample, the existence of these metal-poor, distant objects highlight the diversity among galaxies with similar luminosities or stellar masses. With among the lowest $M / L$ ratios known, our sample allows the study of galaxies at the earliest phases of starbursts and opens up the opportunity to explore the IMFs, dust and extinction laws, gas ionizations, and gas outflows and inflows within very low metallicity, moderately high stellar mass, galactic systems, at high redshifts (see Kakazu et al. 2007 and Hu et al. 2009 for another sample of low-metallicity distant galaxies selected with narrow band photometry).
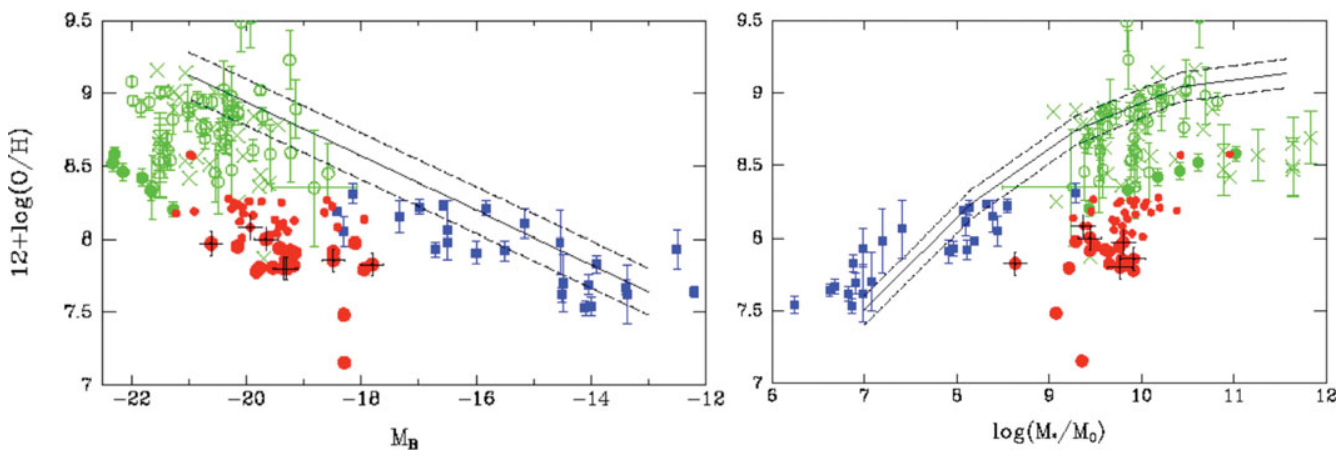

Figure 2. (Left) Gas-phase metallicity $([\mathrm{O} / \mathrm{H}])$ versus luminosity $\left(\mathrm{M}_{B}\right)$ relation $(\mathrm{LZR})$. The solid line with $1 \sigma$ errors (dashed lines) is the mean trend for SDSS galaxies; local dwarf galaxies are solid squares. Various high redshift samples are light-shaded open circles. Our distant low-metallicity galaxies selected by detection of [OIII] $4363 \AA$ are dark solid circles (some with "+" if [OII] was missing). Our sample appears 3 to $6 \sigma$ lower in metallicity than the local mean relation. (Right) Same as the left but versus stellar mass $\left(\mathrm{M}_{*} / \mathrm{M}_{\odot}\right)$. The rms of $\log (\mathrm{O} / \mathrm{H})$ is $\sim$ 0.12 dex over 5 decades of mass (Lee et al. 2006). Our sample appears at lower metallicity by 5 to $10 \sigma$ versus the local relation. Details and figures are given by Hoyos et al. (2010).

\subsection{Ubiquitous Gas Outflows of Galaxies at Redshifts $z \sim 1.4$}

The major DEEP2 study of outflows is by Weiner et al. (2009), who stacked all 1400 spectra in which the MgII $2800 \AA$ doublet lines are accessible near $z \sim 1$. 4 . Of these, only about 200 had AEGIS MIPS data and about 120 had AEGIS HST ACS images. The main result is that the MgII absorption is very strong (roughly $55 \%$ in depth), implying that almost all galaxies in the sample have outflows. Substacks confirm that this result was independent of luminosity, color (within the sample), star formation rate, stellar mass, and morphology. Thus common galaxies, like our Milky Way, had winds and yet 
have not all quenched to become red galaxies. The absorption profiles showed a sawtooth shape, with a median of about $250 \mathrm{~km} / \mathrm{s}$ with an extension to $500 \mathrm{~km} / \mathrm{s}$ and as high as $1000 \mathrm{~km} / \mathrm{s}$ for the most massive galaxies. Most of the galaxies in the sample had SFR of $10-100 M_{\odot} /$ yr (i.e. , LIRG's), which match crude estimates of outflow rates of $20 M_{\odot} /$ yr. While others find that mergers are common among ULIRGs and strong starbursts that show winds, only 3 of 120 galaxies in the AEGIS HST sample appear to be merger-like. Thus, mergers are not necessary to produce such winds. The outflow velocities appear higher for galaxies with higher stellar mass or SFR, roughly $\mathrm{V}$ (wind) $\sim S F R^{0.3}$. This behavior is similar to that found for local ULIRG's by Martin (2005) and favors momentum rather than energy driven winds and higher escape velocities. The DEEP2 results imply that massive galaxies, not dwarves, may dominate wind activity and the enrichment of the IGM, and suggest that such massive galaxy winds should be included in models of galaxy formation.

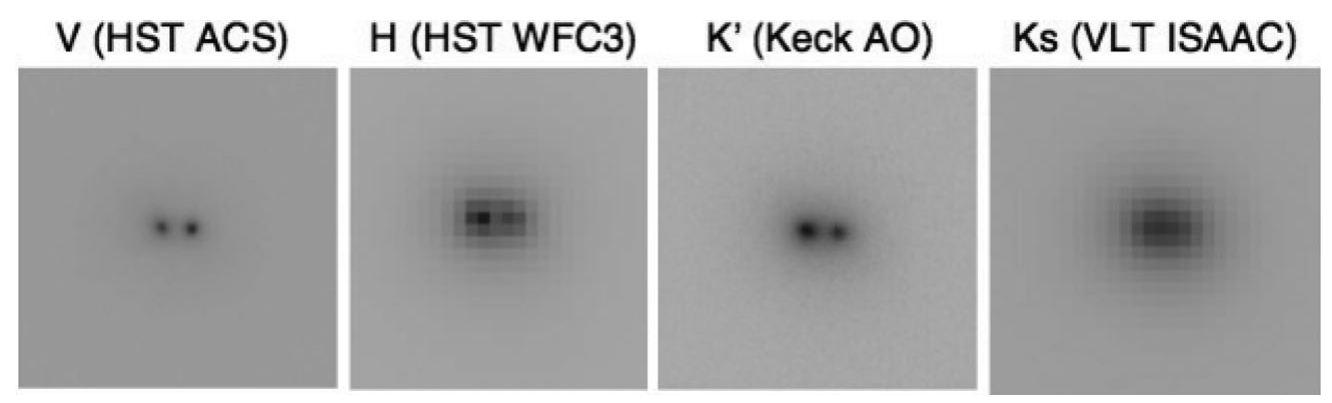

Figure 3. Images of XID 536, a low luminosity Chandra X-ray source at redshift $\mathrm{z}=0.42$ with HST ACS V (F606W), HST WFC3 H (F160W), Keck NIRC2 K', and VLT ISAAC Ks. North is up and East is to the left with the separation of the central two nuclei being about 0.40 arcsec. The V, K', and Ks images (Melbourne et al. 2005) show the excellent sampling (0.04 arcsec pixels) and spatial resolution ( $\sim 0.1$ arcsec FWHM) of the Keck laser guide star AO system. The $\mathrm{H}$ band image is from the HST Early Release Science of the GOODS-S field and is a half-orbit exposure from the WFC3 IR camera with 0.13 arcsec pixels, which under-samples the HST PSF at $\mathrm{H}$ of about 0.16 arcsec. The ISAAC ground-based image had seeing of $\sim 0.6$ arcsec.

\subsection{Optical - Near IR Colors of Distant Galaxy Nuclei}

To date, CATS has obtained AO data for about 5 square arcmin with the Keck NIRC2 camera in GOODS, COSMOS, GEMS, and EGS. One Chandra X-ray galaxy in GOODS$\mathrm{S}$ was found to have two red nuclei (see Fig. 3) for which the 4 filter HST ACS photometry in the optical matched either old, red stars or dusty, reddened, younger stars. The AO K-band photometry provided the critical discriminant - both were red due to old age, not dust. Thus we are witnessing a "dry merger" in progress (Melbourne et al. 2005).

\section{Summary and Future}

DEEP2, AEGIS, and CATS, all using Keck, affirm the potential for stellar population studies of distant galaxies by providing:

1) data to $z \sim 1^{+}$when galaxies were assembling and developing their mature forms;

2) large numbers useful for rare targets and stacking of spectra and images;

3) a multitude of star formation rate measures on different timescales;

4) gas ionization conditions and gas and stellar metallicities;

5) stellar ages and formation histories; 6) stellar and dynamical masses;

7) inflow gas and outflow wind signatures; 
8) deep and rich information on relevant factors that affect stellar populations, such as presence of AGN, mergers, clustering as links to halo mass, environment, etc.

9) with AO, similar information, but for subcomponents of a few galaxies.

The pace of progress on distant galaxy stellar population studies continues to accelerate. For our major survey regions over the next five years, we expect to complete adding the ultra-deep IRAC data from the warm Spitzer mission, more redshifts and spectra, especially from the multiobject near-IR spectrograph on Keck (MOSFIRE), as well as significant data from Herschel, HST WFC3, SCUBA2, and EVLA. For smaller samples, JWST and the next generation AO on Keck will add high resolution images and spectra of distant galaxy bulges, thick and thin disks, bars, super star clusters, AGN hosts, supernovae and gamma-ray bursts, gravitational lenses, etc. A key advance will occur when the next generation of 20-m to 40-m ground optical-IR telescopes becomes reality.

\section{Acknowledgements}

CATS was supported by the NSF Science and Technology CfAO, managed by UC Santa Cruz under cooperative agreement No. AST-9876783. The DEEP team thanks the NSF Science \& Technology CfPA for initiating and supporting the project and NSF, NASA, Quantum, and Sun for funding and equipment. The DEEP, AEGIS, and CATS teams thank Keck and UC Observatories/Lick and their dedicated staff for years of help with DEIMOS and LGS AO and the Hawaiian people for use of their sacred mountain. DCK thanks K. Lai and J. Melbourne for help with the figures.

\section{References}

Ammons, A. M. et al. 2009, AJ, 137, 470

Conselice, C. J. et al. 2005, ApJ, 628, 160

Covington, M. et al. 2010, ApJ, in press

Davis, M. et al. 2007, ApJ, 660, L1

Erb, D. et al. 2006, ApJ, 644, 813

Flores, H. et al. 2006, AA, 455, 107

Glassman, T. M. et al. 2002, ApJ, 581, 865

Hoyos, C. et al. 2005, ApJ, 635, L21

Hoyos, C. et al. 2010, ApJ, in preparation

$\mathrm{Hu}$, E. et al. 2009, ApJ, 698, 2014

Kakazu, Y. et al. 2007, ApJ, 668, 853

Kassin, S. et al. 2007, ApJ, 660, L35

Kunth, D. \& Ostlin, G. 2000, $A \& A R, 10,1$

Larkin, J. E. et al. 2000, PASP, 112, 1526

Lee, H. et al. 2006, ApJ, 647, 970

Liang, Y. C. et al. 2006, AA, 447,113

Martin, C. L. 2005, ApJ, 621, 227

Melbourne, J., et al. 2005, ApJ, 625, L27

Melbourne, J. et al. 2008, AJ, 135, 1207

Newman, J. A. et al. 2010, ApJ, in preparation

Savaglio, S. et al. 2005, ApJ, 635, 260

Shapley, A. E. et al. 2005, ApJ, 635, 1006

Steinbring, E. et al. 2004, ApJS, 155, 15

Steinbring, E. et al. 2008, AJ, 136, 1523

Tremonti, C. A. et al. 2004, ApJ, 613, 898

Vogt, N. P. et al. 2005, ApJS, 159, 41

Weiner, B. J. et al. 2009, ApJ, 692, 187

Wizinowich, P. et al. 2006, PASP, 118, 297 